\title{
Neues zur Biografie von Franz Carl Hiemer (1768-1822)
}

\author{
Von Hildegard Krösche
}

Am 9. August 2018 jährte sich die Geburt des schwäbischen Dichters und Malers Franz Carl Hiemer (Abb.1) zum 250. Mal. Heute noch bekannt ist er für seine Zusammenarbeit mit Carl Maria von Weber (1786-1826): Er lieferte Libretti-Bearbeitungen zu den Opern „Silvana“ (1810) und „Abu Hassan“ (1811) und den Liedtext „Schlaf Herzenssöhnchen“ (1810). Darüber hinaus fertigte er für das Stuttgarter Hoftheater zahlreiche Übersetzungen weiterer Libretti und Schauspiele, vor allem aus dem Französischen und Italienischen. Geradezu berühmt jedoch ist seine Pastellzeichnung (1792) des jungen Friedrich Hölderlin (1770-1843) aus dessen Tübinger Studienzeit, die sich heute im Deutschen Literaturarchiv in Marbach befindet (Abb.2). Dieser 250. Geburtstag soll Anlass sein, einige neue Erkenntnisse zu seinem Lebenslauf, Werk und Tod darzustellen. Nach dem umfangreichen Aufsatz von Rudolf Krauss aus dem Jahr 1906', der auch Quellen in Privatbesitz heranziehen konnte, die heute nur zu einem kleinen Teil in der Württembergischen Landesbibliothek aufbewahrt werden ${ }^{2}$ und ansonsten als verschollen gelten müssen, hat zuletzt Werner Dukex im Jahr 2004 Biografie, Werkverzeichnis und Literatur zusammengestellt ${ }^{3}$.

${ }^{1}$ Rudolf Krauss, Aus Franz Karl Hiemers Leben, in: Württembergische Vierteljahreshefte für Landesgeschichte N.F. 15 (1906) S. 572-598.

2 Württembergische Landesbibliothek Stuttgart (künftig: WLB), Cod. hist. quart. 395; die Schenkung der Briefe wurde im Dezember 1906 von Frl. Cappeyne van de Cappello im Haag durch Vermittlung von H. Finanzrat Teuffel in Stuttgart vermittelt.

3 Werner Dukeк, Er soll „viel Gaaben haben ... “ Der Dichter Franz Carl Hiemer (17681822) aus Rottenacker, in: Musik in Baden-Württemberg 11 (2004) S.149-160; Ders., Ein Leben für die Kunst. Der Autor, Maler und Schauspieler Franz Carl Hiemer, in: Blätter des Schwäbischen Albvereins 109 (2003) S.17; Ders., „Wer spricht schon vom Dichter Hiemer“. Ein Künstlerleben um 1800, in: Schwäbische Heimat 37 (1986) S.303-311. 
Nicht, wie verschiedentlich vermutet, auf besondere Empfehlung fand der junge Hiemer im Alter von 10 Jahren Aufnahme in die Hohe Karlsschule in Stuttgart, sondern eine zufällige Begegnung im Park der damals noch im Bau befindlichen Sommerresidenz des Herzogs Karl Eugen (1728-1793) in Hohenheim bestimmte seine weitere Ausbildung. Eigentlich hätte er nach dem Griechisch- und LateinUnterricht durch den Vater Theologie studieren und in Fortführung der Familientradition Pfarrer werden sollen. Das ergibt sich aus einem handschriftlich überlieferten Lebenslauf seines Bruders Philipp Hiemer (1770-1814). Dieser zwei Jahre jüngere Bruder war zu derselben Zeit wie Hölderlin im Tübinger Stift, und durch ihn kam sein Bruder Carl in Kontakt mit dessen Freundeskreis ${ }^{4}$. Nach dem Theologiestudium ist er zunächst Vikar bei seinem erkrankten Vater Eberhard Friedrich Hiemer (1740-1795) in Oberboihingen gewesen ${ }^{5}$ und wanderte nach dessen Tod zusammen mit seinem Bruder Benedikt (geb. 1771) über St. Petersburg in die evangelischen Kolonien im Wolgagebiet aus. Durch die Vermittlung der Brüdergemeinde in Herrnhut war er zunächst von 1796 bis 1804 Pfarrer in Galka/Ust'Kulalinka, anschließend von 1804 bis zu seinem Tod 1814 in Grimm/Lesnoj Karamyš, beide Gemeinden im Gouvernement Saratov gelegen ${ }^{6}$. Er erwarb sich dort gemeinsam mit zwei anderen Pfarrern vergleichsweise früh Verdienste um die Pockenschutzimpfung ${ }^{7}$. Benedikt Hiemer war nach 1814 zeitweise als Schullehrer in Norka tätig und lebte seit Juni 1816 als Kreisschreiber in Frank/Medvedicko-Krestovoj-Buerak ${ }^{8}$. In dem in Teilen von Philipp Hiemer selbst verfassten, von der Hand seines Bruders Benedikt erhaltenen Lebenslauf (Abb.3) ${ }^{9}$ schildert er auch, wie ihr älterer Bruder Carl in die Stuttgarter Karlsschule aufgenommen wurde:

[...] Ich war der dritte von 5 Brüdern und batte noch drey Schwestern. Nicht mich, sondern den ältesten meiner Brüder, der auch vorzügliche Talente äußerte, hatte mein seliger Vater zum Studium der Theologie bestimmt, ibn bereits nach Stuttgardt zum Examen gebracht und ibn unter die Candidaten aufschreiben

${ }^{4}$ Dukek, Der Dichter (wie Anm. 3) S. 151; Krauss (wie Anm. 1) S. 581 und S. 598 Anm.

5 Krauss (wie Anm. 1) S. 573.

${ }^{6}$ Erik Amburger, Die Pastoren der evangelischen Kirchen Rußlands vom Ende des 16. Jahrhunderts bis 1937. Ein biographisches Lexikon, Erlangen/Lüneburg 1998, S. 44 f., $135,137,350 \mathrm{f}$.

${ }^{7}$ Medicinisch-chirurgische Zeitung, fortgesetzt von Johann Nepomuck EHrHART, Band 2, Salzburg 1809, S. 272 (http://opacplus.bsb-muenchen.de/title/3006568/ft/bsb10084 692? page=9, Aufruf am 12.04.2018). Sie erhielten jeweils eine goldene Dose als kaiserliches Geschenk dafür, dass sie mehrere Tausend Kinder [...] vaccinirt hatten.

${ }^{8}$ Brief vom 15./27.04.1817 von Benedikt Hiemer aus Medvedicko-Krestovoj-Buerak und Norka an einen nicht namentlich angeredeten Bruder (nicht Ferdinand: Carl oder Friedrich), Privatbesitz.

${ }_{9}$ Ebd., mit abschriftlich eingelegtem Lebenslauf von Pastor Graf senior zum Tod von Philipp Hiemer, im ersten Teil von diesem selbst verfasst, Privatbesitz. 
[Die folgenden Abbildungen können aus rechtlichen Gründen online nicht bereitgestellt werden:]

Abb. 1: Franz Carl Hiemer, Ölgemälde von Johann Baptist Seele, 1807 (Vorlage: Privatbesitz).

Abb. 2: Friedrich Hölderlin, Pastellzeichnung von Franz Carl Hiemer, 1792

(Vorlage: Deutsches Literaturarchiv Marbach). 
[Die Abbildung kann aus rechtlichen Gründen online nicht bereitgestellt werden.]

Abb. 3: Lebenslauf von Philipp Hiemer (1770-1814)

(Ausschnitt, Vorlage: Privatbesitz).

laßen, die in die Kloster-Schulen jährlich aufgenommen werden. Bey der Gelegenheit aber kam mein Vater an dem schönen herzoglichen Lustorte Hobenheim vorbey, wolte meinem Bruder die herrlichen Anlagen in der Näbe zeigen, ritt unwissender Weise einen Nebenweg, der verboten war, wurde vom Herzog bemerkt, der auf ibn zugeritten kam, ibn fragte, wer er wäre, und ibm das gnädige Anerbieten machte, meinen Bruder gratis in seine Militärakademie aufzunehmen. Der Herzog behielt ihn auch sogleich zurück, und mein Vater kam allein nach Hause. Dieses war die zufällig scheinende, aber gewiß nicht obne höhere Lenkung gewesene Veranlaßung, daß mein Vater nun mich zum geistlichen Stande widmete. [...]

Der Herzog teilte dem Intendanten der Karlsschule, Obrist von Seeger, seine Entscheidung über die kostenlose Aufnahme Hiemers unverzüglich mit, und dieser wurde an den folgenden beiden Tagen auf seine Leistungen geprüft, ärztlich untersucht und als Eleve aufgenommen ${ }^{10}$. In das von 1770 bis 1793 geführte „Nationalbuch aller Zöglinge“ ist er mit der laufenden Nr.730 als Karlsschüler eingetragen worden ${ }^{11}$. Kurz darauf will der Vater gegenüber dem Intendanten den

10 Krauss (wie Anm.1) S.573; Dukek, Ein Künstlerleben (wie Anm.3) S.304; vgl. Deutsches Literaturarchiv Marbach (künftig: DLA), zu Schiller, Carlsschule Nr.5651, 5654-5656.

11 HStA Stuttgart A 272 Bü 231: Nationalbuch aller Zöglinge, 1770-1793, fol.37, vgl. Werner GebHardt, Die Schüler der Hohen Karlsschule. Ein biographisches Lexikon unter Mitarbeit von Lupold von Lehsten/Frank Raberg, Stuttgart 2011, S. 70, 290f.; dort auch weitere Literaturangaben zur Geschichte der Hohen Karlsschule und der Quellenüberlieferung. 
Charakter seines Sohnes, nunmebrigen Eleven, näher schildern und beginnt den Brief mit den Worten: Noch bin ich ganz eingenommen von der ausserordentlichen Gnade, welche Serenissimus mir auf dem Wege durch eine so unerwartete böchst buldreiche Aufnabme meines Kindes in die Militair-Academie erwiesen. ${ }^{12}$ Gleichzeitig ist er nach dem Erhalt einer übersandten Consignation, was einjeder Eleve bei seiner Aufnabme in die herzogliche Militair-Academie an groß- und kleiner Montirung wie auch andern Erfordernissen ${ }^{13}$ mitbringen müsse, besorgt, dass diese Anschaffungen seine Möglichkeiten übersteigen, und er verweist auf seine Unterhaltung mit dem Herzog: Serenissimus kennen mich, Höchstwelchen ich meine Umstände mündlich und nunmehro auch schriftlich unterthänigst vorgelegt. Dahero haben Höchstdieselbe die mehr als ausserordentliche Gnade mir zugesagt, mein Kind durchaus gratis gnädigst aufzunehmen. ${ }^{14}$ Das Antwortschreiben des Intendanten brachte die erhoffte Bestätigung, dass er von den Anschaffungen solcher Notbwendigkeiten gänzlich befreyet und [...] mithin in diesem Stück obngeachtet des [...] zugekommenen Verzeichnus ganz berubiget bleiben darf ${ }^{15}$.

Die im Lebenslauf erwähnten weiteren Geschwister waren der 1769 geborene Bruder Friedrich sowie Ferdinand (1774-1856), der als Kaufmann in Esslingen lebte ${ }^{16}$, und die Schwestern Dorothea, geb. 1773, Gottliebin (1778-1812) und Johanne (1782-1850). Die mittlere Schwester heiratete 1810 in Hengen den dortigen Pfarrer Carl Christian Gratianus, bei deren nach wenigen Monaten verstorbenem Sohn Franz Carl Hiemer namengebender Pate war ${ }^{17}$. Die beiden anderen Schwestern blieben unverheiratet und lebten offenbar in schwierigen Verhältnissen - sie erbaten vom König Zuwendungen, wie die erhaltenen Dankschreiben aus den Jahren 1819 und 1820 zeigen $^{18}$. Dorothea Hiemer wohnte zumindest zeitweise, nachweislich 1811, bei ihrem Bruder Carl in der Hauptstädter Straße Nr. 22 in Stutt$\operatorname{gart}^{19}$. Ihre Schwester Johanne verbrachte ihre letzten zehn Lebensjahre ab 1840 in

12 DLA, zu Schiller, Carlsschule Nr. 5652.

13 Vorlage: Erfodernissen.

14 DLA, zu Schiller, Carlsschule Nr.5652; eine entsprechende Auflistung ist abgedruckt in: August Friedrich Batz, Beschreibung der Hohen Karls-Schule zu Stuttgart, Stuttgart 1783, Nachdruck Stuttgart 1987, S. 248-251.

15 DLA, zu Schiller, Carlsschule Nr. 5653.

16 Krauss (wie Anm. 1) S. 592; Landeskirchliches Archiv Stuttgart (künftig: LKA), Familienregister Esslingen I, S. 626.

17 LKA, Taufregister Hengen, Bd. 4: Franz Carl Friedrich Gratianus, getauft am 05.08. 1811 in Abwesenheit seines Onkels Franz Carl Hiemers, Oberfinanz-Secretarius in Stuttgardt; nach dem Familienregister, fol. 18 bereits am 11.10.1811 verstorben.

18 HStA Stuttgart E 5 Bü 61 (1819): 22 Gulden, beide Schwestern zusammen; ebd., Bü 62 (1820): 15 Gulden, Dorothée Hiemer.

19 Wegweiser für die königliche Haupt- und Residenz-Stadt Stuttgart, hg. von den vier Kgl. Ober-Polizei-Commissärs, Stuttgart 1811, S.102f. Das Gebäude lag zwischen Brücken- und Kreuzstraße (seit 1957: Dornstraße), vgl. Stadtarchiv Stuttgart, Findbuch 125/1: 
Kirchheim/Teck ${ }^{20}$. Der Bruder Friedrich Hiemer, im Sommer 1792 noch als Kaufmann in Freiburg ${ }^{21}$, war später als Rechnungsführer im Dienst von Obrist Jakob von Wimmer in Wien beschäftigt und für ihn u. a. in Komorn/Ungarn in Holzhandelsgeschäften unterwegs ${ }^{22}$. Ferdinand Hiemer besuchte ihn im Frühjahr 1819 in Wien, als er offenbar schwer erkrankt war, wie aus erhaltenen Familienbriefen an die Brüder hervorgeht. Er ist wohl wie sein Bruder Carl unverheiratet geblieben ${ }^{23}$.

Nach seiner unentgeltlichen Aufnahme in die Karlsschule verbrachte Franz Carl Hiemer die nächsten $121 / 2$ Jahre in dieser Einrichtung und konnte sie erst mit 22 Jahren am 18. Juni 1791 wieder verlassen ${ }^{24}$. Wie nun bekannt ist, hat seine erste Begegnung mit Herzog Karl Eugen in Hohenheim stattgefunden, und dort war es, wo er als Malerzögling der Karlsschule regelmäßig zur Ausmalung der Räume in dem umgebauten Lustschloss herangezogen wurde, eine bei den angehenden Malern sehr unbeliebte Tätigkeit, gegen die jedoch neben Josef Anton Koch $(1768-1839)^{25}$ nur Hiemer laut protestierte ${ }^{26}$. Diese Information ist durch Georg Friedrich Fischer (1769-1841) ${ }^{27}$, einen engen Vertrauten aus der Karlsschulzeit ${ }^{28}$, überliefert - der Nachruf auf den Maler Koch ist zwar lediglich mit F. unterzeichnet, aber durch seine weiteren Angaben, dass er selbst bis 1790 Karlsschüler war, und zudem seit der Bearbeitung des Koch-Nachlasses ist er sicher zu identifizieren $^{29}$. In Fischers Urteil über den 17 Jahre zuvor verstorbenen Franz Carl Hiemer scheint noch die alte Verbundenheit zu einem Akademiekameraden mitzuschwin-

Hauptamt - Straßenbenennungen 1811-1991. Im Jahr 1800 wohnte Hiemer, als Schauspieler (Akteur) aufgeführt, in der Hausnummer 1173 im Lazarethgässle, die 1811 als Lazarettstraße Nr. 383 bezeichnet ist.

${ }^{20}$ LKA, Familienregister Kirchheim/Teck I, S. 865.

21 Krauss (wie Anm. 1) S. 581.

22 Brief vom 18.08.1810 aus Wien an seinen Bruder Ferdinand, Privatbesitz; noch 1817 ist er in Komorn nachweisbar, vgl. Jakob Glatz, Nachrichten über die Feyer des dritten Jubelfestes der Reformation in den sämmtlichen kaiserl. königl. Oesterreichischen Staaten im Jahre 1817, Wien 1818, S. VII (https://opacplus.bsb-muenchen.de/Vta2/bsb10451125/ bsb:BV001598407?page=7, Aufruf am 12.04.2018).

${ }^{23}$ Brief vom 18.08.1810 von Friedrich Hiemer in Wien an Ferdinand, Privatbesitz: Carl und ich werden schon als Hagenstolzen enden müssen.

${ }^{24}$ HStA Stuttgart A 272 Bü 217; vgl. Dukek, Der Dichter (wie Anm.3) S. 151; Krauss (wie Anm. 1) S. 580.

${ }^{25}$ Gebhardt (wie Anm.11) S. 89, 335.

${ }^{26}$ Krauss (wie Anm.1) S. 577.

27 Gebhardt (wie Anm. 11) S. 78, 235.

${ }^{28}$ Er war es, dem einer von drei Briefen Franz Carl Hiemers galt, als er mit 16 Jahren einen Fluchtversuch aus der Karlsschule unternommen hatte, Krauss (wie Anm.1) S.576, vgl. DLA, zu Schiller, Carlsschule Nr. 5659: Herrn Fischer in der herzoglichen Carls-HohenSchule.

29 Axel Kunn, Revolutionsbegeisterung an der Hohen Carlsschule. Ein Bericht, Stuttgart-Bad Cannstatt 1989, S.13-16 mit Hinweis auf Ernst JafFé, Joseph Anton Koch. Sein Leben und sein Schaffen, Innsbruck 1905, S. 12. 
gen, der [...] übrigens als gemüthlicher Dichter und trefflicher Mensch noch bei Vielen in gutem Andenken stebt. ${ }^{30}$

II.

Nach dem ersten Hinweis im Nachruf in der Allgemeinen Literatur-Zeitung vom Jahr 1823, dass Franz Carl Hiemer Soldat war, heißt es 1839 konkreter, er sei Offizier gewesen ${ }^{31}$. Dies bestätigt ein Aktenfund zum Jahr 1812, als ein Streit um Hiemers Anwesenheit in der Schauspielerloge des Stuttgarter Hoftheaters entstanden war. In diesem Zusammenhang schildert Hiemer selbst, er habe sein Ehrenwort gegeben, die Loge unverzüglich zu verlassen ${ }^{32}$. Der beteiligte Platzkontrolleur, Billetabnehmer Weiß, zitiert Hiemer: Er wolle herausgehen, er gebe darauf sein Ehrenwort, er sey Officier gewesen und man werde ibm doch auf sein Ehrenwort glauben ${ }^{33}$. Schon Krauss vermutete, es könnte eine Offiziersstelle bei einem Kreisregiment gewesen $\operatorname{sein}^{34}$. Und tatsächlich erscheint in den Anciennitätslisten des Schwäbischen Kreises beim Württembergischen Infanterieregiment mit dem Eintrittsdatum 16. Januar 1795 ein Fähnrich Hiemer in der Kompagnie des Hauptmanns von Neubronn ${ }^{35}$.

Im Verlauf des Ersten Koalitionskrieges gegen Frankreich standen die Regimenter des Schwäbischen Kreises, darunter das 5. Kreis-Infanterie-Regiment unter Herzog Friedrich Eugen, dessen Sammelplatz bis 1796 Weil der Stadt war, als Teil der Reichstruppen bei Kehl, um unter der Führung von Feldzeugmeister von Stain als Teil der österreichischen Oberrheinarmee den Rheinübergang zu sichern ${ }^{36}$. Nach dem gelungenen Durchbruch der französischen Truppen und dem von Württemberg und auch dem Schwäbischen Kreis geschlossenen Waffenstillstand im Sommer 1796 zählte Hiemer zu den übercompleten Offizieren des 3. Bataillons und erhielt im Dezember desselben Jahres eine monatliche Friedensgage von

30 (Georg Friedrich) F(ISCHER), Joseph Anton Koch, in: Europa, Chronik der gebildeten Welt, hg. von August Lewald, Band 2, Stuttgart 1839, S.289-308, hier S.292 (http:// opacplus.bsb-muenchen.de/title/6388526/ft/bsb10612132?page=5, Aufruf am 12.04.2018).

31 Ebd.

32 StAL E 18 I Bü 133, Schreiben Hiemers vom 19. 05. 1812 sowie ebd., Bü 350, Schreiben Hiemers vom 20.05.1812.

33 Ebd., Bü 350, Konzept von Hofrat Döring vom 19.05.1812; vgl. auch sein Schreiben von demselben Tag, ebd.

34 Krauss (wie Anm. 1) S. $580 \mathrm{f}$.

${ }^{35}$ HStA Stuttgart C 14 Bü 24 a, z. T. ist der Name auch als von Hiemer angegeben. Im Adreß-Buch 1796, S. 101 ist Hiemer ebenfalls als Fähnrich aufgeführt.

36 Winfried Dotzauer, Die deutschen Reichskreise (1383-1806). Geschichte und Aktenedition, Wiesbaden 1998, hier: Der Schwäbische Kreis, S.142-179; Hans-Joachim HARDER, Militärgeschichtliches Handbuch Baden-Württemberg, hg. vom Militärgeschichtlichen Forschungsamt, Stuttgart u. a. 1987, besonders S.34-47. 
17 Gulden ${ }^{37}$. Seine nachgesuchte Entlassung hat der Fähnrich Hiemer am 11. November 1797 erhalten ${ }^{38}$.

Leider sind die Offizierspatente des Schwäbischen Kreises nicht vollständig überliefert, und in den erhaltenen Militärakten ist außer dem Namen Hiemer weder Vorname noch Herkunft vermerkt. Doch in die bislang bekannte Biografie lassen sich die Angaben gut einfügen: Demnach wäre Franz Carl Hiemer unmittelbar nach dem Tod seines am 7. Januar 1795 verstorbenen Vaters, der sich so ausdrücklich gegen eine Karriere seines Sohnes als Schauspieler ausgesprochen hatte ${ }^{39}$, Offizier geworden, so dass er sich vielleicht noch auf dessen Rat oder aus der nun noch dringenderen Notwendigkeit einer eigenen Versorgung heraus zu diesem Schritt entschloss - erst 1799 ist er dann als Hofschauspieler nachweisbar ${ }^{40}$. Er hatte durch seine Ausbildung (und die dabei geknüpften Kontakte) auf der Stuttgarter Karlsschule und seine vergleichsweise stattliche Körpergröße sicherlich nicht die schlechtesten Voraussetzungen für den Dienst bei den Kreistruppen ${ }^{41}$. Mit dem Eintritt in ein Kreisregiment folgte er im Übrigen auch dem Vorbild seines Großvaters Franz Carl Seefels (1712-1778), der ebenfalls als Fähnrich beim Württembergischen Kreiskontingent zu Fuß gedient hatte ${ }^{42}$.

Mit dieser knapp dreijährigen Militärzeit - und der seit Januar 1798 nachweisbaren Tätigkeit für das „Schwäbische Industriekontor“ in Heilbronn ${ }^{43}$ - lässt sich die bisherige Lücke in der Biografie, die durch das von KRAuss festgestellte Fehlen der Korrespondenz für die Jahre 1795 bis 1798 bedingt war ${ }^{44}$, schließen. Die Erfahrungen des Kriegsdienstes hat Franz Carl Hiemer offenbar in seinem 1795 erschienenen „Kriegslied“ (Schön ist's unterm freien Himmel ...) verarbeitet, das von Johann

37 HStA Stuttgart C 14 Bü 42, Anciennetäts- und Rangliste von Januar 1797 und Bü 655a, Schreiben vom 09.12.1796. Im Herzoglich Wirtembergischen Addreß-Buch auf das Jahr 1797, S. 60 erfolgt die Nennung Hiemers unter den Second-Lieutenants, das entspricht einer in einem Gutachten vom 24.12.1796 erwähnten Gleichstellung überzähliger Offiziere: die übercomplete Fähnrichs und Fabnenjunkers sind in dieser Hinsicht anzuseben, als ob sie in gleichem Grad mit dem Second-Lieutenant der Regimenter stehen; HStA Stuttgart C 14 Bü 655 a.

38 Ebd., Bü 655 a.

39 WLB Cod. hist. quart. 395, fol.54-55, Brief vom 29.10.1790; Krauss (wie Anm.1) S. 580 .

40 Krauss (wie Anm. 1) S. 586.

${ }^{41}$ DLA, zu Schiller, Carlsschule Nr.5649; vgl. Abb. bei Dukek, Ein Künstlerleben (wie Anm.3): Der Personalbogen aus der Karlsschule gibt seine Körpergröße zuletzt, mit ca. 19 bis 21 Jahren, mit 6 Fuß 2 Strich an, was etwa $172 \mathrm{~cm}$ entspricht.

${ }^{42}$ Hanns Wolfgang Rath, Regina, die schwäbische Geistesmutter. Reprint der 1. Auflage Ludwigsburg/Leipzig 1927, neu bearbeitet, ergänzt und erweitert durch Hansmartin DECKer-Hauff, Limburg an der Lahn 1981, S. 60 mit Anm. 142; Walther Pfeilsticker, Neues Württembergisches Dienerbuch, 3 Bände, Stuttgart 1957-1974, Band 2, 33341.

${ }^{43}$ WLB Cod. hist. quart. $447 \mathrm{r}$ Nr.6, Brief von Hiemer im Auftrag des Industriekontors an Ludwig Neuffer vom 10.01.1798; Krauss (wie Anm.1) S. 585.

44 Krauss (wie Anm. 1) S. 585. 
Christian Gottlob Eidenbenz vertont wurde ${ }^{45}$. Ähnlich wie das spätere „Wiegenlied“, dessen Melodie von Carl Maria von Weber stammt, fand es weite Verbreitung und Eingang in verschiedene Lieder-Sammlungen ${ }^{46}$.

\section{III.}

Hiemers Tod am 15. November 1822 wurde in der Ausgabe des Schwäbischen Merkurs vom 17. November knapp in drei Zeilen angezeigt. Einen Tag später, in der Ausgabe vom 19. November, erschien die Anzeige seines Bruders Ferdinand für Verwandte und Freunde ${ }^{47}$. Die Beerdigung fand am 18. November in Stuttgart statt. Die Grabrede, die auf Veranlassung seiner Freunde bei der Hof- und Kanzlei-Buchdruckerei Gebrüder Mäntler in Stuttgart gedruckt wurde (Abb.4), hielt Repetent M. Hoffmann ${ }^{48}$ :

Ueberraschend schnell für Alle, die ibren Werth kannten, entwand sich ibrer irrdischen Hülle die Seele, über deren Abschied von der Erde wir hier tranern. Wer hätte wobl diesem Manne bei der Heiterkeit seines Gemüths, bei so vielem Lebensmuth nur dieses Alter geschätzt? - Wer hätte wobl geglaubt, daß er dem Kreise der Geschwister und Freunde, die mit der vollen Liebe ibrer Herzen an ihm bingen, jetzt schon entrückt werden würde? Wer hätte vermuthet, daß der Mann von diesem kräftigen Körperbau mit dem 54 sten Jabre das Ende seines Lebens erreichen sollte? - Des Herrn Gedanken und Wege sind nicht unsre Gedanken und unsre Wege! - so müssen wir auch dießmal wieder sprechen - gebengt unter die Hand des Schicksals, doch voll Glaubens an die Güte seiner Beschlüsse.

Wie sehr berubigt uns insbesondere die Gewißheit, daß es immer ein stiller Wunsch des Entschlafenen war, schnell und leicht, obne die Qualen einer langen Krankheit, in eine andre Welt übergeführt zu werden! - Ja, sein Loos fiel ihm, wie er es wünschte: freundlich schloß ibm der Todesengel das Ange und fübrte ibn, obne daß er ibre Schrecken vernabm, an den Pforten seines Reiches vorüber; er befand sich in einer andern Welt, obne die Beschwerden des Uebergangs kennen gelernt zu haben. Und wenn wir in der Art seines Todes schon die || Gnade Gottes gegen ibn ahnen - o so würden wir an seinem Grabe noch viel mehr uns trösten können, wenn

45 Taschenbuch für Freunde des Gesanges, Stuttgart 1795, S. $131 \mathrm{f}$.

${ }^{46}$ Vgl. beispielsweise die Nachweise bei August Heinrich Hoffmann von FallersLEBEn, Unsere volkstümlichen Lieder, hg. und neu bearbeitet von Karl Hermann PRAHL, Nachdruck der 4. Auflage Leipzig 1900, Hildesheim 1966, Nr. 1009.

47 Text bei Krauss (wie Anm.1) S. 592.

${ }^{48}$ Das ist wohl M. Ernst Friedrich Gottlieb Hoffmann (1789-1866), der als Repetent im Stift Tübingen 1813-1815 und gleichzeitig als Hofmeister des Herzogs Alexander von Württemberg 1813-1823 nachgewiesen ist, so Christian SigEL, Das evangelische Württemberg. 2. Hauptteil: Generalmagisterbuch nach den Personalakten im Landeskirchlichen Archiv in Stuttgart, Bd. 12, S. 911. 


\section{Fede}

\section{a $m$ (s) $r$ b e}

\section{$\operatorname{ses}$}

\section{Şert! frtanß Ceart Siemtr,}

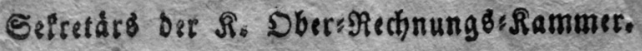

Cebalten

Den 18. RoDember 1823

bon De का

\section{Şerrm Repetenten M. Şofimam.}

Abb. 4: Leichenpredigt auf Franz Carl Hiemer, 1822 (Vorlage: Privatbesitz).

uns vergönnt wäre, zu schauen, wie er drüben, im Lande der Vergeltung, empfangen wurde. Dort, du Vollendeter! wirst Du angeschrieben gefunden haben die stillen Werke der Liebe, die Du, geleitet von Deinem guten Herzen, hienieden übtest! Dort werden die tausend kleinen Gefälligkeiten des Lebens, mit denen Du Alle, die Dich berührten, Dir verbandest, geschätzt seyn als die Ausflüsse eines woblwollenden Gemüthes: dort werden alle die Opfer, die Du für das Glück Vieler, die Dir näher oder entfernter angehörten, so willig brachtest, anerkannt seyn als die Wirkungen einer Seele, die von dem Gebote durchdrungen ist: „Liebe deinen Nächsten wie dich selbst!"

Und uns, meine Lieben, sollte an seinem Grabe der Glaube: „ibm wird vergolten" - nicht trösten können? Wir sollten mit unsrem Blicke hängen bleiben an diesem Gefilde des Todes, und blos an dem Vergangenen haftend, blos an uns denkend - uns nicht erbeben können über die Schmerzen dieser Stunde? Nein! Wir wissen: die Liebe fordert ibre Opfer! Und so webe es den Gemüther der Freunde 
thun mag, den Theuren nun nimmer zu seben, den immer Bereitwilligen, so es zu belfen galt, nun entbebren zu müssen - sie berubigen sich über das, was sie verloren haben, mit dem Glauben: ibm ist ja nun völlig wobl! nimmer ausgesetzt den Wechseln des Irrdischen, geschützt vor allen Stürmen der Erde - genießt er den Frieden einer $\|$ bessern Welt und erndet aus der Hand des allgütigen Gottes, was er hienieden in seinem Sinne gesäet hat.

Einen freundlichen Dank rufen ibm nach ${ }^{49}$ so Viele, denen er durch seine vielseitigen Talente genützt, mit seiner Geschicklichkeit gedient, die er durch die Gaben seines heiteren, offenen Gemüthes erfreut hat! Es verdient wohl vorzügliches Lob, daß Er - durch seine Lebensverhältnisse so vielfach angesprochen, vermöge seiner verschiedenen Fertigkeiten auf die mannichfaltigste Weise thätig - doch stets mit dem beharrlichsten Fleiße seiner Berufsgeschäfte wartete, und auch daran darf gewiß mit besonderem Rubme erinnert werden, daß er nach dem Zeugnisse von Personen, die ibn kannten, bei aller Munterkeit seiner Laune doch stets in Wort und That von Allem entfernt blieb, was gute Sitten beleidigt.

„Selig sind, die reinen Herzens sind!" möchte dieser Spruch, den Du, Hingegangener! in Deinem Leben sonach nicht überhörtest, immer mehr auch der unsrige werden! Dein schneller Abschied von Welt und Leben, - dieser Schauplatz der Verwesung - die hier zerstreuten traurigen Reste von Menschen, die auch einst, wie wir, eine Weile anf dieser Oberfläche gelebt, gelitten und sich gefreut habendiese verödete Natur, in der Alles zum Winterschlafe sich anschickt - die Asche so mancher Blume, die noch vor wenigen Monden lachend hier stand - dieser Bäume gesenktes Haupt - o alles das ruft laut genug uns zu: du mußt vergehen, o Mensch! mit einem Theile deines Wesens der Natur angellhörend blübst und wächst $d u$, wie ibre Erzeugnisse alle, aber dann gebt dein Weg, wie der ibre, abwärts und $d u$ verschwindest, um einer andern Erscheinung Platz zu machen! - Nun wenn denn auch wir unerbittlich dem Gesetze der Vergänglichkeit folgen müssen, wenn wir oft genug daran gemahnt werden - o so lasset uns doch unaufgehalten für die Rettung des Theiles an uns arbeiten, der-nicht irdischer Abkunft-auch nicht den Gesetzen des Irdischen erliegt. Wie thöricht, alle Kraft, alle Bestrebungen im Leben nur darauf zu richten, um es dem Theile an uns recht wobl zu machen, für den wir nur ein Paar Jabre etwas, und dann eine ganze Ewigkeit nichts mehr thun können, der uns - nur für diese Welt geliehen - eigentlich gar nicht angehört! - Wie thöricht, die Entwicklung und Bildung jener Seite an uns zu verabsäumen, auf deren Zustand beim Ausgange aus dem Leben alles ankommt, für die wir nichts mehr thun können, wenn die Nacht kommt, wo Niemand mebr wirken kann! - Nein, auch dieses Grab wollen wir nicht verlassen, obne neue Entschlüsse für das Eine, was Noth thut, gefaßt zu haben, und an das letzte Lebewohl, das wir dem vielgeschätzten Freunde nachrufen, knüpfe sich der Vorsatz, unsern Sinn und unser Leben immer mehr durchdringen zu lassen von jenem Geiste, der bei all seinem Wirken die

\footnotetext{
49 Vorlage: noch.
} 
Gesetze des Reiches Gottes voll Ehrfurcht stets vor Augen hat. Denn nur für sie, die Einen Weg - den Weg der Heiligung und Liebe - gewandelt sind, giebt es ein Wiederseben! Amen.

Die Rede wurde von den vierstimmigen Gesängen Sanfte Rube, tiefer Frieden / Herrschet in der Gräber Nacht! [...] und Rube sanft in stillem Gottesfrieden! I Schlummre zu dem bessern Leben hin! [...] eingerahmt, deren Text dort ebenfalls abgedruckt ist.

Über den finanziellen Nachlass Hiemers gibt ein Brief aus Russland des Bruders Benedikt an seine Schwester Dorothea indirekt Auskunft. Den noch lebenden Geschwistern, das waren zumindest Benedikt, Ferdinand, Dorothea und Johanna, bzw. den Nachkommen des verstorbenen Philipp fiel ein Erbteil von jeweils 37 Gulden 35 Kreuzer zu; ihre Mutter war bereits 1810 in Nürtingen gestorben ${ }^{50}$. Im August 1824 schreibt Benedikt Hiemer aus der Kolonie Norka:

Deinen Brief vom 8. Juni vorigen Jabrs habe ich im December erbalten. Nach einem 10-jäbrigen Stillschweigen kamen mir Familien-Todesnachrichten nicht unerwartet, überraschend war mir die Ansicht deines Briefs und daß ich noch in diesem Leben von dir und einen Monat später auch von Bruder Ferdinand Briefe erbielt, indem ich hierauf bereits Verzicht gethan hatte. Den Schmerz, einen treuen Bruder zu verlieren, lernte ich bey dem Tode des seligen Philip in vollem Maaß kennen - Gott ist mehr als menschliche Hülfe, wovon Ihr, seitdem Euch Eure Stüze durch den Tod des guten Bruders Carl entzogen worden, gewiß nun mehr redende Beweise haben werdet. Du schreibst mir, daß von dem Nachlaß des seligen Bruders Carl den Erben des verstorbenen Bruders Philipp 37 Gulden 35 Kreuzer und mir ebensoviel erblich gehöre. Die Schwägerin, des Bruders Philip Witwe, und Tochter ist schon $1 \mathrm{Jahr}$ bey mir, auch seit $1 / 4 \mathrm{Jahr}$ ihr jüngster Sobn Johann, der morgen wieder zurück nach Sarepta reißen will - diesen habe ich auszugsweise deinen Brief vorgelesen und von ibnen beyliegende Erklärung erhalten, daß sie ibren Erbtheil von dem seligen Carl dir und der Schwester Johanna schenken wollen, welchem Beyspiel ich mit Vergnügen folgte und um Eu[er wi]llen wünschte, daß es mehr seyn möchte $[\ldots] .{ }^{51}$

${ }^{50}$ LKA, Sterberegister Nürtingen, Bd.23, Nr. 38: 17. Juli 1810.

51 Brief vom 15./27.08.1824 an Dorothea Hiemer, Privatbesitz. 